\title{
A New Method for the Self-Tuning Control of Nonminimum Phase Discrete-Time Systems in the Presence of Disturbances
}

\author{
Member Jianming Lu (Chiba University) \\ Member Muhammad Shafiq (Chiba University) \\ Nonmember Takashi Yahagi (Chiba University)
}

\begin{abstract}
We present a new method for the self-tuning control (STC) of nonminimum phase discrete-time systems based on the pole-zero placement. We show that the effect of unstable zeros on the output. can be canceled approximately. The long division method is used to decompose a polynomial into a stable polynomial and an unstable one. Finally, the results of computer simulation are presented to illustrate the effectiveness of the proposed method.
\end{abstract}

Keywords: Self-tuning control, Nonminimum phase systems, Discrete-time systems

\section{Introduction}

The usual adaptive control techniques cannot be applied to nonminimum phase systems. It has long been known that such plants are not easy to control [1]. There exists fundamental limitation to the controlled performance, because boundedness of all signals is not assured due to the unstable pole-zero cancellation in the adaptive control systems [2].

Pole assignment techniques have been proposed to avoid the unstable pole-zero cancellation. However, these methods assign only the poles in the closed-loop system and so their response characteristics are not as good as that can be obtained from a reference model type [3]. Although self-tuning controller based on polezero placement has been proposed to overcome this problem, yet unstable zeros of the plant cannot be canceled [4]. Using these techniques unstable zeros must also be zeros of the closed-loop system. Furthermore, these techniques cannot be applied to systems with disturbances [5].

To overcome this problem, we propose a new method for the adaptive control of nonminimum phase system such that the effect of the unstable zeros on the closedloop can be canceled approximately and disturbances can be decoupled. In this method we use long division method to decompose the numerator polynomial of the plant into stable and unstable polynomials.

Furthermore, in the feedforward controller we include the approximate inverse system of the unstable part of the numerator polynomial of the plant such that the effect of unstable zeros on the plant output can be canceled approximately and there exists no unstable pole-zero cancellation in the system. We find this approximate inverse system by using binomial expansion. The method is suitable to use in practical applications.

\section{Problem Statement}

Consider the following SISO discrete-time system

$$
A(z) y(t)=z^{-d} B(z) u(t)+w(t)
$$

where

$$
A(z)=1+\sum_{i=1}^{n} a_{i} z^{-i}, \quad B(z)=\sum_{i=0}^{m} b_{i} z^{-i} .
$$

Furthermore, $d$ is a delay of the system, $A(z)$ and $B(z)$ are coprime polynomials, $b_{0} \neq 0$ and $z$ is a difference operator, $u(t)$ is the input and $y(t)$ is the output and $w(t)$ is the disturbance expressed by

$$
w(t)=w_{0}+w_{1} t+\cdots+w_{k} t^{k}
$$

Moreover, the plant is a nonminimum phase system and the coefficients of $A(z)$ and $B(z)$ are unknown.

In these circumstances, we show that a controller can be designed such that the closed-loop system is stable and that the transfer function of the system, as a whole, is given by

$$
W(z) \simeq z^{-d} \frac{B_{m}(z)}{A_{m}(z)},
$$

where $A_{m}(z)$ and $B_{m}(z)$ are arbitrarily chosen stable polynomials of degree $\rho$ and $\mu$, respectively. In this situation the input $r(t)$ to the system having transfer function $z^{-d} B_{m}(z) / A_{m}(z)$ yields the output $y(t)$.

\section{Design Method for Known Systems without Disturbances}

In this case, the plant can be given by 


$$
y(t)=z^{-d} \frac{B(z)}{A(z)} u(t) .
$$

The unstable zeros of a plant cannot be canceled by the controller, because in this situation there will exist unstable oscillations in the system. This means the zeros of the closed-loop transfer function of a nonminimum phase system cannot be assigned arbitrarily, i.e. the closed-loop must contain the unstable zeros of the plant.

Let us consider the case in which $B(z)$ has an unstable zero at $-1 / \beta$. As we cannot cancel this zero, a closed-loop can be designed such that $[4]$

$$
y(t)=z^{-d}\left(\beta+z^{-1}\right) \frac{B_{m}(z)}{A_{m}(z)} r(t),
$$

where $|\beta|<1$, and $A_{m}(z)$ and $B_{m}(z)$ are arbitrarily chosen stable polynomials satisfying the conditions given in Sect. 2.

The aim of this paper is to cancel the effect of the unstable zeros on the output $y(t)$ approximately. To cancel the effect of this unstable zero, we consider the following binomial approximation

$$
\left(\beta+z^{-1}\right)^{-1} \simeq z-\beta z^{2}+\beta^{2} z^{3}-\cdots-(-1)^{L} \beta^{L-1} z^{L} .
$$

Now, including this approximation in the feedforward controller, the output can be given by

$$
\begin{aligned}
y(t)= & z^{-d}\left(z-\beta z^{2}+\beta^{2} z^{3}-\cdots-(-1)^{L} \beta^{L-1} z^{L}\right) \\
& \times\left(\beta+z^{-1}\right) \frac{B_{m}(z)}{A_{m}(z)} r(t)
\end{aligned}
$$

where

$$
\begin{aligned}
& \left(z-\beta z^{2}+\beta^{2} z^{3}-\cdots-(-1)^{L} \beta^{L-1} z^{L}\right)\left(\beta+z^{-1}\right) \\
& \quad=1+(-1)^{L+1} \beta^{L} z^{L} .
\end{aligned}
$$

Therefore, the output can be expressed by

$$
\begin{aligned}
y(t)= & z^{-d}\left(1+(-1)^{L+1} \beta^{L} z^{L}\right) \frac{B_{m}(z)}{A_{m}(z)} r(t) \\
= & z^{-d} \frac{B_{m}(z)}{A_{m}(z)} r(t) \\
& +(-1)^{L+1} \beta^{L} z^{L-d} \frac{B_{m}(z)}{A_{m}(z)} r(t),
\end{aligned}
$$

where $|\beta|<1$, so by selecting sufficiently large positive integer $L$ we can make $\beta^{L}$ small to the desired level. For $\beta=0.7$ and $L=20$, we obtain $\beta^{L}=7.9792 \times 10^{-4}$. Thus

$$
\left|y_{1}(t)\right|<<|y(t)|
$$

where

$$
y_{1}(t)=(-1)^{L+1} \beta^{L} z^{L-d} \frac{B_{m}(z)}{A_{m}(z)} r(t) .
$$

Then the output can be written as

$$
y(t) \simeq z^{-d} \frac{B_{m}(z)}{A_{m}(z)} r(t) .
$$

Let us consider the case in which $B(z)$ has complex unstable zeros at $-1 / \beta$ and $-1 / \bar{\beta}$. A closed-loop can be designed such that

$$
y(t)=z^{-d}\left(\beta+z^{-1}\right)\left(\bar{\beta}+z^{-1}\right) \frac{B_{m}(z)}{A_{m}(z)} r(t),
$$

where $\bar{\beta}$ is a complex conjugate of $\beta$. Again using binomial approximation, we may write the following expression

$$
\begin{aligned}
& \left(\beta+z^{-1}\right)^{-1}\left(\bar{\beta}+z^{-1}\right)^{-1} \simeq \\
& \quad\left(z-\beta z^{2}+\beta^{2} z^{3}-\cdots-(-1)^{L} \beta^{L-1} z^{L}\right) \\
& \quad\left(z-\bar{\beta} z^{2}+\bar{\beta}^{2} z^{3}-\cdots-(-1)^{L} \bar{\beta}^{L-1} z^{L}\right) .
\end{aligned}
$$

It can be easily shown that the coefficients of the polynomial $\left(z-\beta z^{2}+\beta^{2} z^{3}-\cdots-(-1)^{L} \beta^{L-1} z^{L}\right)\left(z-\bar{\beta} z^{2}+\right.$ $\left.\bar{\beta}^{2} z^{3}-\cdots-(-1)^{L} \bar{\beta}^{L-1} z^{L}\right)$ are real. In this case

$$
\begin{aligned}
& \left(z-\beta z^{2}+\beta^{2} z^{3}-\cdots-(-1)^{L} \beta^{L-1} z^{L}\right)\left(\beta+z^{-1}\right) \\
& \times\left(z-\bar{\beta} z^{2}+\bar{\beta}^{2} z^{3}-\cdots-(-1)^{L} \bar{\beta}^{L-1} z^{L}\right)\left(\bar{\beta}+z^{-1}\right) \\
& =\left(1+(-1)^{L+1} \beta^{L} z^{L}\right)\left(1+(-1)^{L+1} \bar{\beta}^{L} z^{L}\right) \\
& =1+(-1)^{L+1}\left(\beta^{L}+\bar{\beta}^{L}\right) z^{L}+(-1)^{2 L+2}(\beta \bar{\beta})^{L} z^{2 L} .
\end{aligned}
$$

As $\beta$ and $\bar{\beta}$ are complex numbers, we may write $\beta=|\beta| e^{j \theta}$ and $\bar{\beta}=|\beta| e^{-j \theta}$. Here $\theta$ represents argument of $\beta$ and $-\theta$ denotes argument of $\bar{\beta}$. It can be shown that $\left(\beta^{L}+\bar{\beta}^{L}\right)$ and $(\beta \bar{\beta})^{L}$ are real. Therefore, the output can be given by

$$
\begin{gathered}
y(t)=z^{-d} \frac{B_{m}(z)}{A_{m}(z)} r(t)+(-1)^{L+1}\left(\beta^{L}+\bar{\beta}^{L}\right) z^{L-d} \\
\times \frac{B_{m}(z)}{A_{m}(z)} r(t)+(-1)^{2 L+2}(\beta \bar{\beta})^{L} z^{2 L-d} \frac{B_{m}(z)}{A_{m}(z)} r(t),(15)
\end{gathered}
$$

where $\left|\left(\beta^{L}+\bar{\beta}^{L}\right)\right|<<1$ and $\left|(\beta \bar{\beta})^{L}\right|<<1$. So, once again the output can be expressed as

$$
y(t) \simeq z^{-d} \frac{B_{m}(z)}{A_{m}(z)} r(t)
$$

Keeping this idea in mind we may design the closed-loop as follows.

If we choose an asymptotically stable polynomial $T(z)$ then there exist unique polynomials $R(z)$ and $S(z)$, which satisfy $[4,6,7]$

$$
A(z) R(z)+z^{-d} B(z) S(z)=A_{m}(z) T(z),
$$

where $\operatorname{deg} R(z)=\rho+\operatorname{deg} T(z)-n$ and $\operatorname{deg} S(z)=n-1$.

Let us define the control input $u(t)$ by

$$
R(z) u(t)=T(z) r_{1}(t)-S(z) y(t),
$$

where

$$
r_{1}(t)=\frac{B_{m}(z) M(z)}{B^{+}(z)} r(t) .
$$

In eq. (19), $B^{+}(z)$ is a factor of polynomial $B(z)$, and $B^{+}(z)$ has all roots inside the unit disc. Generally, 
it may be complicated to decompose the polynomial $B(z)$ into a stable polynomial $B^{+}(z)$ and an unstable polynomial $B^{-}(z)$. Then long division method [5] can be used to find a stable polynomial

$$
B^{+}(z)=b_{0}\left(1+p_{1} z^{-1}+p_{2} z^{-2}+\cdots+p_{\xi} z^{-\xi}\right)
$$

and an unstable polynomial

$$
B^{-}(z)=1+q_{1} z^{-1}+q_{2} z^{-2}+\cdots+q_{\psi} z^{-\psi} .
$$

Moreover,

$$
\begin{aligned}
M(z) & =\left(z-v_{1} z^{2}+v_{1}^{2} z^{3}-\cdots-(-1)^{L} v_{1}^{L-1} z^{L}\right) \\
& \times\left(z-v_{2} z^{2}+v_{2}^{2} z^{3}-\cdots-(-1)^{L} v_{2}^{L-1} z^{L}\right) \cdots \\
& \times\left(z-v_{\psi} z^{2}+v_{\psi}^{2} z^{3}-\cdots-(-1)^{L} v_{\psi}^{L-1} z^{L}\right),
\end{aligned}
$$

where

$$
\begin{gathered}
\left(v_{1}+z^{-1}\right)^{-1} \simeq z-v_{1} z^{2}+\cdots-(-1)^{L} v_{1}^{L-1} z^{L} \\
\left(v_{2}+z^{-1}\right)^{-1} \simeq z-v_{2} z^{2}+\cdots-(-1)^{L} v_{2}^{L-1} z^{L} \\
\vdots \\
\left(v_{\psi}+z^{-1}\right)^{-1} \simeq z-v_{\psi} z^{2}+\cdots-(-1)^{L} v_{\psi}^{L-1} z^{L}
\end{gathered}
$$

and $\left(v_{1}+z^{-1}\right),\left(v_{2}+z^{-1}\right), \cdots,\left(v_{\psi}+z^{-1}\right)$ are the factors of the unstable polynomial

$$
B^{-}(z)=1+q_{1} z^{-1}+q_{2} z^{-2}+\cdots+q_{\psi} z^{-\psi} .
$$

It is important to note here that if all the roots of polynomial $B^{-}(z)$ lie out side the unit disc then $\left|v_{i}\right|<1$ $(i=1,2, \cdots, n)$ hold. It is easy to check the accuracy of the approximations given in eqs. $(4) \sim(6)$ by inspecting the following relations

$$
\begin{aligned}
&\left(v_{1}+z^{-1}\right)\left(z-v_{1} z^{2}+v_{1}^{2} z^{3}-\right.\left.\cdots-(-1)^{L} v_{1}^{L-1} z^{L}\right) \\
&=1+(-1)^{L+1} v_{1}^{L} z^{L} \\
&\left(v_{2}+z^{-1}\right)\left(z-v_{2} z^{2}+v_{2}^{2} z^{3}-\cdots-(-1)^{L} v_{1}^{L-1} z^{L}\right) \\
&= 1+(-1)^{L+1} v_{2}^{L} z^{L} \\
& \vdots \\
&\left(v_{\psi}+z^{-1}\right)\left(z-v_{\psi} z^{2}+v_{\psi}^{2} z^{3}-\cdots-(-1)^{L} v_{\psi}^{L-1} z^{L}\right) \\
&=1+(-1)^{L+1} v_{\psi}^{L} z^{L}
\end{aligned}
$$

Now, $y(t)$ can be given by

$$
y(t)=z^{-d} \frac{B_{m}(z)}{A_{m}(z)} r(t)+z^{-d} P(z) \frac{B_{m}(z)}{A_{m}(z)} r(t),
$$

where

$$
\begin{aligned}
P(z)= & -1+\left(1+(-1)^{L+1} \eta_{1} z^{L}\right)\left(1+(-1)^{L+1} \eta_{2} z^{L}\right) \\
& \times \cdots \times\left(1+(-1)^{L+1} \eta_{\psi} z^{L}\right) \\
= & (-1)^{L+1}\left(\eta_{1}+\eta_{2}+\cdots+\eta_{\psi}\right) z^{L}+(-1)^{2 L+2} \\
& \left(\eta_{1} \eta_{2}+\eta_{1} \eta_{3}+\cdots+\eta_{1} \eta_{\psi}+\cdots\right. \\
& \left.+\eta_{\psi-1} \eta_{\psi}\right) z^{2 L}+\cdots \\
& +(-1)^{\psi L+\psi}\left(\eta_{1} \eta_{2} \cdots \eta_{\psi}\right) z^{\psi L}
\end{aligned}
$$

and

$$
\eta_{1}=v_{1}^{L}, \quad \eta_{2}=v_{2}^{L}, \cdots, \quad \eta_{\psi}=v_{\psi}^{L} .
$$

It is important to note here that $\left|v_{i}\right|<1 \quad(i=$ $1,2, \cdots, \psi)$. Therefore, if we choose a large positive integer $L$ then $\left|\eta_{i}\right| \ll<1$. Thus, it is possible to make the coefficients of $P(z)$ sufficiently small such that

$$
\left|z^{-d} \frac{B_{m}(z)}{A_{m}(z)} r(t)\right|>>\left|z^{-d} P(z) \frac{B_{m}(z)}{A_{m}(z)} r(t)\right| .
$$

Therefore, we may state

$$
y(t) \simeq z^{-d} \frac{B_{m}(z)}{A_{m}(z)} r(t) .
$$

\section{Example 1}

Let us consider the case in which $v_{1}=0.5$ and $v_{2}=$ 0.6 and $L=20$. Then, $\eta_{1}=1.9073 \times 10^{-6}$ and $\eta_{2}=$ $6.0936 \times 10^{-5}$. The absolute values of the coefficients of $P(z)$ are given as

$$
\left|\eta_{1}+\eta_{2}\right|=6.2843 \times 10^{-5}, \quad\left|\eta_{1} \eta_{2}\right|=1.1623 \times 10^{-10} .
$$

Fig. 1 shows the relationship between $L$ and coefficients of $P(z)$ in this example.

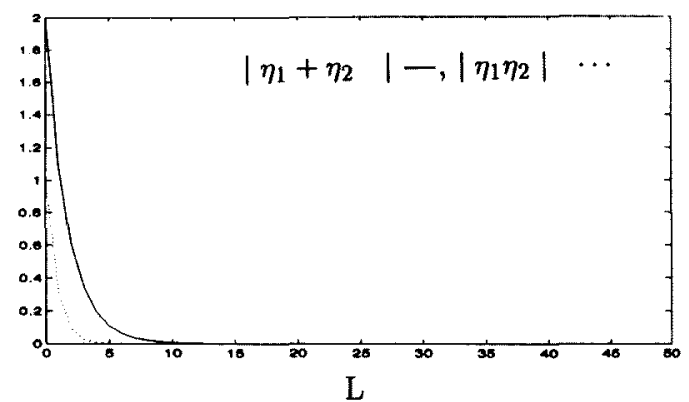

Fig. 1 relationship between $L$ and coefficients of $P(z)$

Therefore, the output $y(t)$ of the plant given by eq (1) can be made to follow some output $y_{m}(t)$ approximately. The output $y_{m}(t)$ is obtained from a system whose transfer function is $z^{-d} B_{m}(z) / A_{m}(z)$ and is excited by input $r(t)$. This transfer function must obey the conditions given in Sect. 2 .

\section{Design Method for Known Systems with Disturbances}

Here we consider the plant with disturbance given in eq. (1). The disturbance expressed in eq. (2) can be eliminated by introducing a polynomial $D(z)$ such that

$$
D(z) w(t)=0 \text {. }
$$

This objective can be achieved by choosing $D(z)$ as

$$
D(z)=\left(1-z^{-1}\right)^{k+1}
$$

Now, equation (17) can be rewritten for the plant described in eq. (1) as

$$
A(z) D(z) R(z)+z^{-d} B(z) S(z)=A_{m}(z) T(z),
$$


where $\operatorname{deg} R(z)=\rho+\operatorname{deg} T(z)-n-k-1$ and $\operatorname{deg} S(z)=$ $n-1$.

Let the input $u(t)$ satisfy the following relation

$$
N(z) u(t)=T(z) r_{1}(t)-S(z) y(t)
$$

where

$$
\begin{aligned}
M(z)= & \left(z-v_{1} z^{2}+v_{1}^{2} z^{3}-\cdots-(-1)^{L} v_{1}^{L-1} z^{L}\right) \\
& \times\left(z-v_{2} z^{2}+v_{2}^{2} z^{3}-\cdots-(-1)^{L} v_{2}^{L-1} z^{L}\right) \cdots \\
& \times\left(z-v_{\psi} z^{2}+v_{\psi}^{2} z^{3}-\cdots-(-1)^{L} v_{\psi}^{L-1} z^{L}\right),
\end{aligned}
$$

and

$$
N(z)=D(z) R(z)
$$

and $r_{1}(t)$ is the same as given in eq. (19).

Now, using eqs. (1), (33) (36) we obtain

$$
\begin{aligned}
y(t)= & z^{-d} \frac{B_{m}(z)}{A_{m}(z)} r(t)+z^{-d} P(z) \frac{B_{m}(z)}{A_{m}(z)} r(t) \\
& +\frac{D(z) R(z)}{T(z) A_{m}(z)} w(t) .
\end{aligned}
$$

Again, adopting the same procedure as in Sect. $3, y(t)$ can approximately be given by

$$
y(t) \simeq z^{-d} \frac{B_{m}(z)}{A_{m}(z)} r(t)+\frac{D(z) R(z)}{T(z) A_{m}(z)} w(t) .
$$

As $A_{m}(z)$ and $T(z)$ are stable polynomials, using eq. (31) the effect of the disturbances can be decoupled from the plant output. The transfer function of the closedloop from $r(t)$ to $y(t)$ can be approximately given by $z^{-d} B_{m}(z) / A_{m}(z)$. In the proposed method, main result is that the closed-loop system is stable even in the case of nonminimum phase system and the effect of the unstable zeros on the output can be canceled approximately and the effect of the disturbances can be decoupled.

\section{Design Method for Unknown Systems}

If the coefficients of the plant given by eq. (1) are unknown, then the parameters of the plant can be estimated as follows. Let us introduce a filter to eliminate the disturbances such that the filtered input $u_{f}(t)$ and output $y_{f}(t)$ are defined by

$$
\begin{aligned}
& F(z) y_{f}(t)=D(z) y(t), \\
& F(z) u_{f}(t)=D(z) u(t),
\end{aligned}
$$

where $F(z)$ is a Hurwitz polynomial.

Using eqs. (31), (39) and (40), the system equation can expressed by

$$
y_{f}(t)=-\sum_{i=1}^{n} a_{i} z^{-i} y_{f}(t)+\sum_{j=0}^{m} b_{j} z^{-d-j} u_{f}(t)=\alpha^{T} X_{f}(t)
$$

where $T$ denotes the transpose, and

$$
\alpha^{T}=\left[a_{1}, a_{2}, \cdots, a_{n}, b_{0}, b_{1}, \cdots, b_{m}\right]
$$

and

$$
\begin{aligned}
X_{f}^{T}(t)= & {\left[-y_{f}(t-1), \cdots,-y_{f}(t-n),\right.} \\
& \left.u_{f}(t-d), \cdots, u_{f}(t-d-m)\right] .
\end{aligned}
$$

The vector $\alpha$ represents the unknown parameters of the plant to be estimated, and $X(t)$ is a vector consisting of input and output signals

If the vector $X_{f}(t)$ is persistently exciting, then the parameter adjustment law which ensures that the estimated parameters will converge to their true values can be given by $[8,9]$

$$
\begin{aligned}
\hat{\alpha}(t)= & \hat{\alpha}(t-1) \\
& -\frac{\Gamma(t-1) X_{f}(t)\left[\hat{\alpha}^{T}(t-1) X_{f}(t)-y_{f}(t)\right]}{1+X_{f}^{T}(t) \Gamma(t-1) X_{f}(t)}, \\
\Gamma(t)= & \frac{1}{\sigma}\left[\Gamma(t-1)-\frac{\lambda \Gamma(t-1) X_{f}(t) X_{f}^{T}(t) \Gamma(t-1)}{\sigma+\lambda X_{f}^{T}(t) \Gamma(t-1) X_{f}(t)}\right], \\
\Gamma(0)= & \delta I, \quad \delta>0,
\end{aligned}
$$

where $0<\sigma \leq 1$ and $0<\lambda<2$. by

The control input $u(t)$ in the adaptive case is given

$$
u(t)=\frac{T(z)}{\hat{N}(z)} r_{1}(t)-\frac{\hat{S}(z)}{\hat{N}(z)} y(t),
$$

and

$$
r_{1}(t)=\frac{B_{m}(z) \hat{M}(z)}{\hat{B}^{+}(z)} r(t),
$$

where $\hat{N}(z)$ and $\hat{S}(z)$ are the estimates of $N(z)$ and $S(z)$, respectively. Furthermore, $\hat{S}(z)$ and $\hat{R}(z)$ are obtained from eq. (33) by using the estimates of $A(z)$ and $B(z)$ at time $t$. Then $\hat{M}(z)$ is calculated from eq. (35), and $\hat{N}(z)$ from eq. (36).

\section{Simulation Results}

In the following examples, we will fix the parameters $\sigma, \lambda$ and $\delta$ as

$$
\sigma=0.9, \quad \lambda=1.98 \text { and } \delta=4000 \text {. }
$$

Example 2.

Consider the following plant

$$
\begin{array}{r}
A(z)=1+1.2 z^{-1}+2 z^{-2}+0.1 z^{-3}, \\
B(z)=1-3.3 z^{-1}+1.45 z^{-2}+2.975 z^{-3} .
\end{array}
$$

In this example, we assume $d=1$. The transfer function of this plant has a stable pole at -0.0515 and two unstable poles at $-0.5742 \pm 1.2693 i$. The plant has 
two unstable zero at $2 \pm 0.5 i$ and a stable zero -0.7 . The desired transfer function of the closed-loop is

$$
W(z)=z^{-d} \frac{B_{m}(z)}{A_{m}(z)}=z^{-d},
$$

where $A_{m}(z)=B_{m}(z)=1$.

The simulation results illustrated in Fig. 2 show that the output of the system converges to the output and the estimated parameters converge to their true values. Moreover, it can be seen from Fig. 2 that $y_{1}(t)=$ $z^{-d}\left[P(z) B_{m}(z) / A_{m}(z)\right] r(t)$ is very small as compared to $y(t)$.

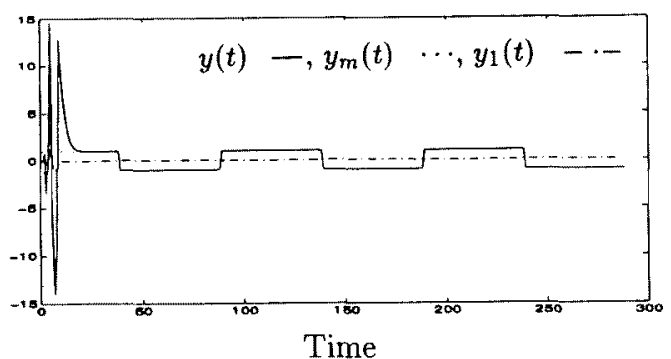

(a)

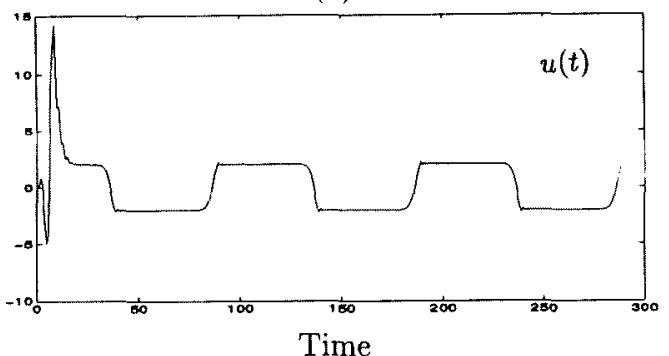

(b)

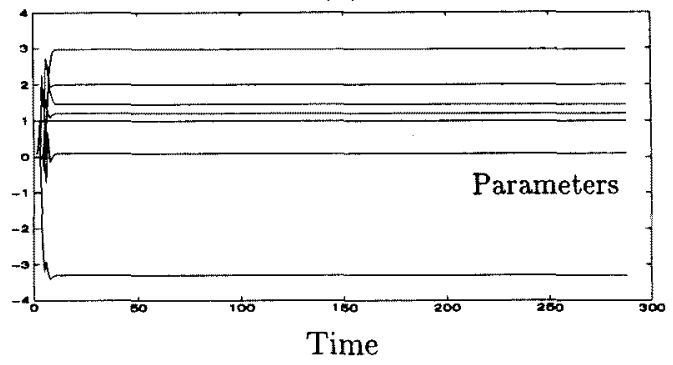

(c)

Fig. 2 Simulation results for example 2.

Example 3.

Let us consider another example in which the plant is described by

$$
\begin{aligned}
& A(z)=1+0.2 z^{-1}+3 z^{-2}+0.1 z^{-3} \\
& B(z)=1+2.7 z^{-1}+1.5 z^{-2}+0.2 z^{-3}
\end{aligned}
$$

This plant has two unstable poles at $-0.0833 \pm 1.728 i$ and one stable pole at -0.0334 , and has one unstable zero at -2 and two stable zeros at -0.5 and -0.2 . Moreover, the plant parameters vary during the operation to

$$
A(z)=1+0.8 z^{-1}+0.9 z^{-2}+0.9 z^{-3},
$$

$$
B(z)=1+3.5 z^{-1}+1.54 z^{-2}+0.12 z^{-3} .
$$

In this example, we assume $A_{m}=B_{m}=1$ and $d=$ 1. In this case, the plant poles are given by $0.0524 \pm$ $0.9960 i$ and 0.9047 and zeros by $-3,-0.4$ and -0.1 . The simulation results are depicted in Fig. 3.

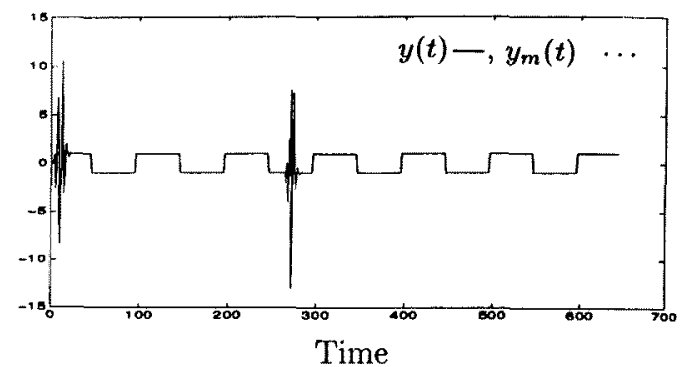

(a)

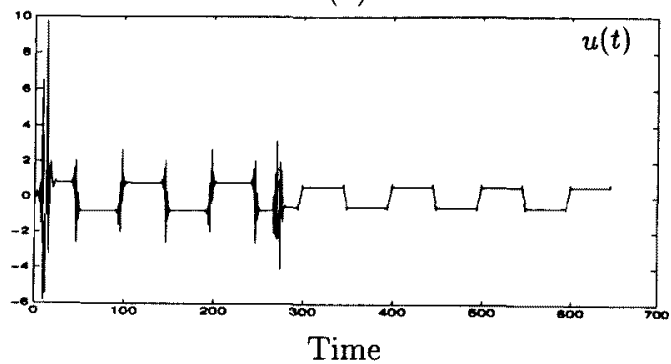

(b)

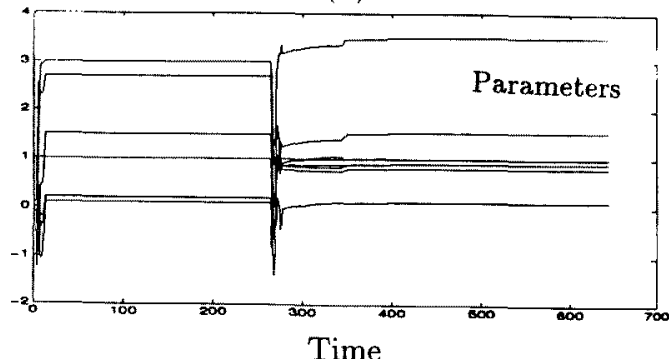

(c)

Fig. 3 Simulation results for example 3 .

It can be realized from Fig. 3 again that output of the plant converges to the desired output and the estimated parameters converges to their true values even if the plant parameters vary during the operation.

Example 4.

Now, we consider the plant with disturbances described by

$$
\begin{aligned}
& A(z)=1+0.1 z^{-1}+0.31 z^{-2}-0.411 z^{-3}, \\
& B(z)=1+1.4 z^{-1}-0.86 z^{-2}+0.68 z^{-3} .
\end{aligned}
$$

This plant is stable having one unstable zero at -2 and two stable zeros. Again we consider the case in which $A_{m}(z)=B_{m}(z)=1$ and $d=1$. Let the disturbance be 
given by

$$
w(t)=\left\{\begin{array}{cc}
0 & 0<t \leq 90 \\
1 & 91<t \leq 220 \\
0 & 221<t \leq 350 \\
4 & 351<t .
\end{array}\right.
$$

Let us choose $F(z)=1-0.5 z^{-1}$ and $D(z)=1-z^{-1}$. The simulation results are shown in Fig. 4. It can be seen from Fig. 4 that the output of the plant converges to the output, and estimated parameters converges to their true values. Moreover, disturbances are decoupled from the plant output. The input and output of the plant do not diverge.

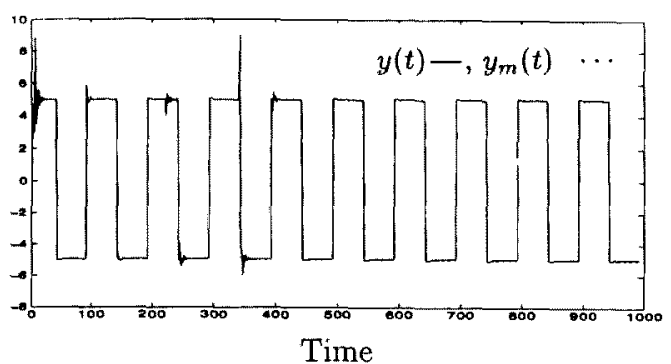

(a)

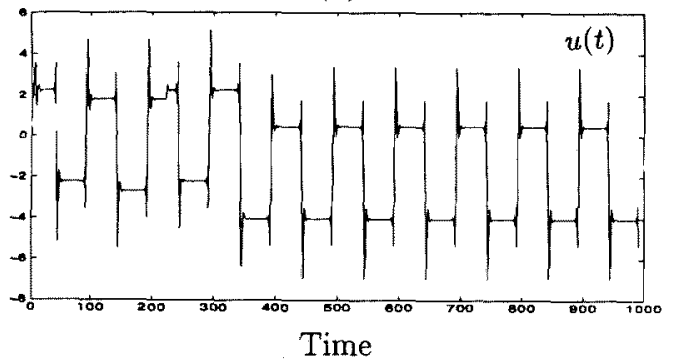

(b)

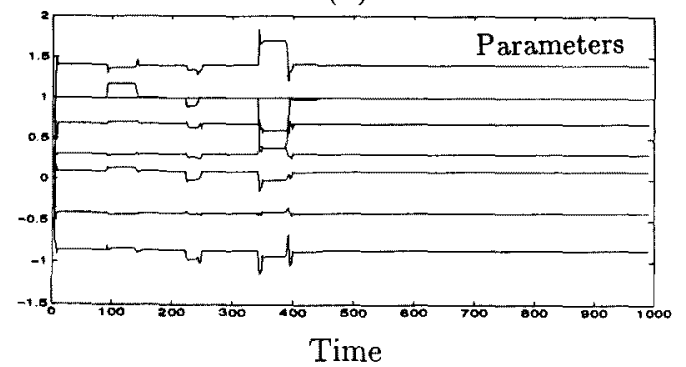

(c)

Fig. 4 Simulation results for example 4.

\section{Conclusions}

We have proposed a new method for STC of nonminimum phase SISO discrete-time systems based on the pole-zero placement technique. It has been shown that the effect of the unstable zeros on the output can be approximately canceled. The method works well even if there exist disturbances.

Long division method is used to decompose an unstable polynomial into stable and unstable polynomials. Furthermore, estimated parameters converge to the plant parameters even if the plant parameters slowly vary during the operation. It has been concluded that the method is useful for the self-tuning control of nonminimum phase systems.

\section{Appendix}

The long division method [10] is used to decompose a polynomial into stable and unstable polynomials.

We consider the case when the polynomial $B(z)$ has at least one zero outside the unit disc. In this case, the power expansion of $1 / B(z)$ is not convergent and its coefficients will eventually become very large. If the long division method is used, at least one zero of $B(z)$ outside the unit disc is eliminated at each long division. The inversion process is terminated whenever the remainder has all zeros inside the unit disc or when the remainder is reduced to a constant. Hence at every long division step, the number of terms in the remainder (the next divisor) is reduced at least by one. Finally, the remainder has all zeros inside the unit disc.

The polynomial $B(z)$ is given by

$$
B(z)=b_{0}+b_{1} z^{-1}+b_{2} z^{-2}+\cdots+b_{m} z^{-m} .
$$

Let the polynomial $B(z)$ be decomposed as

$$
B(z)=B^{+}(z) B^{-}(z),
$$

where

$$
\begin{gathered}
B^{+}(z)=b_{0}\left(1+p_{1} z^{-1}+p_{2} z^{-2}+\cdots+p_{\xi} z^{-\xi}\right), \\
B^{-}(z)=1+q_{1} z^{-1}+q_{2} z^{-2}+\cdots+q_{\psi} z^{-\psi} .
\end{gathered}
$$

and $m=\xi+\psi$. We assume that $B^{+}(z)$ is a stable polynomial, whereas $B^{-}(z)$ is an unstable polynomial. As stated above, by using the long division method, we can find a stable polynomial $B^{+}(z)$ only by simple divisions. There is no need to find the roots of $B(z)=0$.

From eq. (A.1) and eq. (A.2), we obtain

$$
\begin{gathered}
b_{0}\left(1+b_{1}^{\prime} z^{-1}+b_{2}^{\prime} z^{-2}+\cdots+b_{m}^{\prime} z^{-m}\right) \\
=b_{0}\left(1+p_{1} z^{-1}+p_{2} z^{-2}+\cdots+p_{\xi} z^{-\xi}\right) \\
\quad \times\left(1+q_{1} z^{-1}+q_{2} z^{-2}+\cdots+q_{\psi} z^{-\psi}\right)
\end{gathered}
$$

From eq. (A.3), we can calculate the coefficients of the unstable polynomial $B^{-}(z)$ by

$$
\begin{aligned}
& q_{1}=b_{1}^{\prime}-p_{1}, \\
& q_{2}=b_{2}^{\prime}-p_{2}-q_{1} p_{1}, \\
& \quad \vdots \\
& q_{i}=b_{i}^{\prime}-p_{i}-q_{1} p_{i-1}-\cdots-q_{i-1} p_{1},
\end{aligned}
$$

where $p_{i}=0$ for $i>\xi$.

It should be noted that if the long division method is used, there is no need to calculate the roots of $B(z)=0$, and we can find stable and unstable polynomials. Hence, the method is very effective to decompose a polynomial into two factors. Furthermore, this 
method is particularly advantageous for high-order systems.

(Manuscript received December 4, 1995)

\section{References}

[1] Isermann, R., Lachmann, K. H., and Matko, D., Adaptive control systems, Prentice-Hall International, 1994.

[2] Yahagi, T. and Lu, J. " On self-tuning control of nonminimum phase discrete-time systems using approximate inverse systems, " Journal of Dynamic Systems, Measurement, and Control, Trans. ASME, vol. 115, no. 1, pp. 12-18, Mar. 1993.

[3] Lu, J. and Yahagi, T. " Self-tuning control of nonminimum phase systems based on pole-zero placement using approximate inverse systems, "Elect. letters, vol. 29, No. 1, pp. 90-91, Jan. 1993.

[4] Åström, K. J. and Wittenmark, B., " Self-tuning controllers based on pole-zero placement, "Proc. IEE, vol. 127D, no. 3, pp. 120-130, Mar. 1980.

[5] Lu, J. and Yahagi, T., " New design method for model reference adaptive control for nonminimum phase discrete-time systems with disturbances, " IEICE Trans., vol. J75-A, no. 4, pp. 743-753, Apr. 1992.

[6] Kučera, V. Discrete Linear Control, John Wiley \& Sons, 1979.

[7] Barnett, S., Polynomials and Linear Control Systems, Marcel Dekker, Inc., New York, 1983.

[8] Ljung, L. and Söderström, T., Theory and Practice of Recursive Identification, The MIT. Press, Cambridge, 1983.

[9] Johnson, C. R. Jr., Lectures on Adaptive Parameter Estimation, Prentice-Hall, New Jersey, 1988.

[10] Yahagi, T., Theory of Digital Signal Processing, vol. 2, Corona Pub. Co., Ltd., Tokyo, 1985.

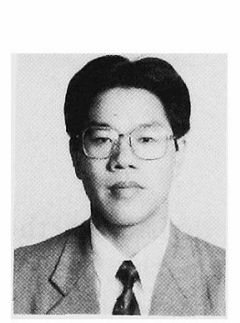

Jianming $\mathbf{L u}$ received the B.E. degree in electrical engineering from the Shan-dong Institute of Mining and Technology, China, in 1983, the M.S., and Ph.D. degrees from Chiba University, Japan, in 1990 and 1993, respectively. In 1993, he joined Chiba University, Chiba, Japan, as an Assistant Professor in the Department of Information and Computer Sciences. His current research interests are in the theory and applications of digital signal processing and control theory. Dr. Lu is a member of IEICE(Japan) and SICE(Japan).

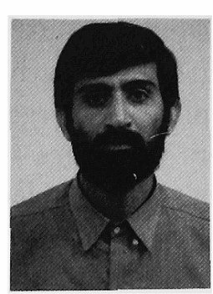

Muhammad Shafiq received the B.E. degree in electronics engineering from NED University, Karachi, Pakistan, in 1989. He joined SES Pakistan, as Assistant Engineer in 1990. From Oct., 1993 to March, 1994, he was a research student at Chiba University. Currently he is completing the Ph.D. degree. He is a member of IEICE(Japan) and a student member of the IEEE(USA). His main research interests are in adaptive control and computer control theory.

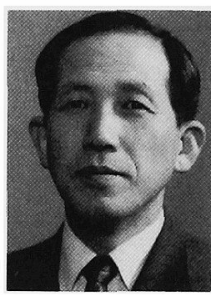

Takashi Yahagi was born in Tokyo, Japan, on Feb. 1, 1943. He received the B.S., M.S., and Ph.D. degrees all in electronic engineering from the Tokyo Institute of Technology Tokyo, Japan, in 1966, 1968, and 1971, respectively. In 1971, he joined Chiba University, Chiba, Japan, as a Lecturer in the Department of Electronic Engineering. From 1974 to 1984 he was an Associate Professor, and in 1984 he was promoted to Professor in the Department of Electrical Engineering. Since 1989 he has been with the Department of Information and Computer Sciences. He was General Chairman of the Second Makuhari International Conference on High Technology: Information Science and Computer Technology, Makuhari, Chiba, Japan, in 1991. His current research interests are in the theory and applications of digital signal processing and other related areas. $\mathrm{He}$ received the Award from the Telecommunications Advancement Foundation in 1989. He is the author of "Theory of Digital Signal Processing, Vols. 1-3"(1985, 1985, 1986), (Corona Pub. Co., Ltd., Tokyo, Japan). He is the Editor of "Digital Signal Processing Library", and also the author of "Digital Signal Processing and Basic Theory" (1996), (Corona Pub. Co., Ltd., Tokyo, Japan). Dr. Yahagi is a member of the IEEE (USA), IEICE(Japan), SICE(Japan), the New York Academy of Sciences (USA), the Institute of Systems, Control and Information Engineers (Japan), the Japan Society of Ultrasonics in Medicine, the Japanese Society of Printing Science and Technology. 